Teresinha de Jesus Noske Rossi

Novas formas de defesa na Era dos Excessos

\author{
Dissertação de Mestrado
}

\author{
Departamento de Psicologia \\ Programa de Pós-Graduação em \\ Psicologia Clínica
}




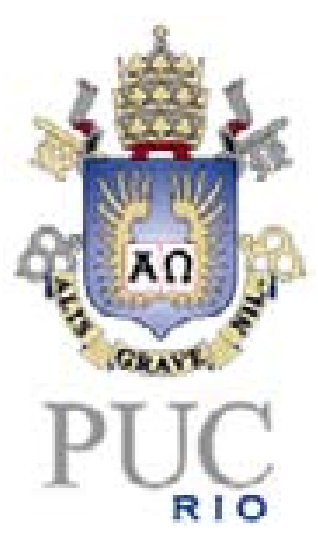

Teresinha de Jesus Noske Rossi

Novas formas de defesa na Era dos

Excessos

Dissertação de Mestrado

Dissertação apresentada como requisito parcial para obtenção do grau de Mestre pelo Programa de Pósgraduação em Psicologia Clínica do Departamento de Psicologia da PUC-Rio.

Orientadora: Ana Maria Nicolaci-da-Costa

Rio de Janeiro

Janeiro de 2004 


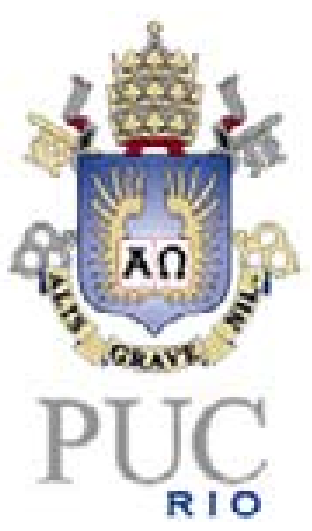

Teresinha de Jesus Noske Rossi

\title{
Novas formas de defesa na Era dos \\ Excessos
}

\begin{abstract}
Dissertação apresentada como requisito parcial para obtenção do grau de Mestre pelo Programa de Pósgraduação em Psicologia Clínica do Departamento de Psicologia da PUC-Rio. Aprovada pela Comissão Examinadora abaixo assinada.
\end{abstract}

Profa Ana Maria Nicolaci-da-Costa

Orientadora

Departamento de Psicologia - PUC-Rio

Profa. Andréa Seixas Magalhães Departamento de Psicologia - PUC-Rio

Profa. Maria Lúcia Rocha Coutinho Instituto de Psicologia - UFRJ

Prof. Paulo Fernando Carneiro de Andrade Coordenador Setorial de Pós-Graduação e Pesquisa do Centro de Teologia e Ciências Humanas - PUC-Rio

Rio de Janeiro, 27 de janeiro de 2004 
Todos os direitos reservados. É proibida a reprodução total ou parcial do trabalho sem autorização da autora, da orientadora e da universidade.

\section{Teresinha de Jesus Noske Rossi}

Graduou-se em Psicologia na Pontifícia Universidade Católica do Rio de Janeiro (PUC-Rio), no ano de 2001, com atuação na área de Psicologia Escolar.

Ficha Catalográfica

Rossi, Teresinha de Jesus Noske

Novas formas de defesa na Era dos excessos / Teresinha de Jesus Noske Rossi ; orientadora: Ana Maria Nicolaci-da-Costa. - Rio de Janeiro : PUC, Departamento de Psicologia, 2004.

142 f. ; $30 \mathrm{~cm}$

Dissertação (mestrado) - Pontifícia Universidade Católica do Rio de Janeiro, Departamento de Psicologia.

Inclui referências bibliográficas.

1. Psicologia - Teses. 2. Comunicação interpessoal. 3. Tecnologias da informação. 4. Internet. 5. Correio eletrônico. 6. Interação. 7. Excessos. 8. Formas de defesa. I. Nicolaci-da-Costa, Ana Maria. II. Pontifícia Universidade Católica do Rio de Janeiro. Departamento de Psicologia. III. Título.

CDD: 150 
Para meu avô Octaviano Rossi (em memória), que me deixou como legado a paixão pela leitura e pelo conhecimento.

Para meus pais, Maura e Filipe, meus avós Jandyra, Wilhelm (em memória) e Therezinha (em memória), meus irmãos Cláudio e Rodrigo e meu sobrinho Erik. Para meu marido Marco. 


\section{Agradecimentos}

Aos meus pais, Luís Filipe Rossi e Maura Rosado Noske pelo suporte, apoio e carinho durante toda minha vida.

Ao meu marido Marco Smilgat por seu amor e dedicação.

À minha orientadora Ana Maria Nicolaci-da-Costa por sua dedicação e colaboração neste trabalho.

Aos meus avós Octaviano Rossi (em memória) e Jandyra da Silveira Rossi, Wilhelm Hermann Noske (em memória) e Maria Therezinha Rosado Machado (em memória).

Aos meus irmãos Claudio e Rodrigo Rossi e ao meu sobrinho Erik Rossi por fazerem parte de minha vida e estarem sempre em meus pensamentos.

Aos meus sogros Maria José e Antônio Marmo Brandão, pelo apoio constante.

Aos meus tios Alexandre e Cláudia.

Aos meus queridos "filhotes" Varg, Celta e Tyr, por tornarem menos árduos os momentos difíceis e compartilharem tanto os bons quantos os maus momentos.

Ao meu querido amigo Alan Aguinaga por me "salvar" inúmeras vezes dos problemas com o computador.

Aos amigos Zezinho, Sheila, Ângela e Marcelo, Luciana, Rodrigo, Filipe, Renato, "Mac" e meu afilhado Derick, Carla e Leandro, Martha, Carola e a todas as outras pessoas maravilhosas que fazem parte de minha vida.

À Terezinha Féres Carneiro e Marise, pela rápida ajuda e dedicação em um momento difícil. 
Aos amigos da PUC Renata Granchi e Ricardo de Castro pelas várias idéias e colaborações neste trabalho.

Às demais amigas do grupo de pesquisa Carla Leitão, Ana Paula Lobão, Érika Falcão, Rosane Abreu, Daniela Romão, Camila e Andréa.

À professora Cláudia Garcia por seus ensinamentos.

À minha querida professora e amiga Mariângela Monteiro.

À PUC-Rio e à vice-reitoria acadêmica pela excelente graduação e por me oferecerem mais esta oportunidade.

À CAPES pelo suporte financeiro durante a execução deste trabalho.

A todos os colaboradores da pesquisa. 


\section{Resumo}

Rossi, Teresinha de Jesus Noske; Nicolaci-da-Costa, Ana Maria. Novas formas de defesa na Era dos Excessos. Rio de Janeiro, 2004. 142p. Dissertação de Mestrado - Departamento de Psicologia, Pontifícia Universidade Católica do Rio de Janeiro.

Este estudo teve como objetivo investigar como os usuários da Internet vêm lidando com o crescente aumento de possibilidades de interação proporcionado pelos novos dispositivos comunicacionais virtuais e, em especial, pelo correio eletrônico. Para tanto, foram realizadas quinze entrevistas de perguntas abertas com homens e mulheres que tivessem no mínimo 25 anos de idade e que já estivessem utilizando a Internet e o correio eletrônico há pelo menos um ano. Após transcrito, o material coletado nessas entrevistas foi analisado através da técnica de análise do discurso. Foi possível concluir que três novos excessos relativos à esfera da comunicação interpessoal já estão fazendo parte do cotidiano desses internautas: o excesso de interação, o excesso de demanda de interação e o excesso de mensagens recebidas diariamente. Foi possível verificar, ainda, que estes usuários vêm buscando diferentes formas de lidar com estes excessos e deles se defender. Entre estas novas formas de defesa encontram-se: a priorização de mensagens urgentes, a abertura de diferentes contas de e-mail com a finalidade de separar diferentes níveis de intimidade, a utilização de uma conta de e-mail extra para evitar informar os endereços pessoais a sites ou pessoas não confiáveis, o rápido apagamento de mensagens não desejadas, a utilização de um momento especial para ler e responder mensagens e, finalmente, o controle - bem como eventual redução - da quantidade de contatos via e-mail.

\section{Palavras-chave}

Comunicação interpessoal, tecnologias da informação, Internet, correio eletrônico, interação, excessos, formas de defesa. 


\section{Abstract}

Rossi, Teresinha de Jesus Noske; Nicolaci-da-Costa, Ana Maria (Advisor). New forms of defense in Overload Age. Rio de Janeiro, 2004. 142p. MSc. Dissertation - Departamento de Psicologia, Pontifícia Universidade Católica do Rio de Janeiro.

This study aimed at investigating how Internet users are dealing with the increase of interaction possibilities brought about by new communication devices and, specially, by the e-mail. An investigation was carried out with fifteen men and women who were at least twenty-five years old and had been using the Internet and e-mail programs for at least a year. An open-ended-question interview was conducted with each of them. After transcribed, the material collected in these interviews was analyzed through discourse analysis techniques. The results of this analysis made it possible to identify the emergence of three types of excess concerning the new forms of interaction: the excess of interaction, the excess of demand for interaction and the excess of incoming messages. These results also made it possible to conclude that these users are using different forms to deal with these excesses and to defend themselves from them. They give priority to urgent messages, open different e-mail accounts in order to separate different levels of intimacy, use at least one extra account so that they do not have to inform their personal address to sites or people they do not trust, quickly delete undesirable messages, use a special moment of the day to read and reply messages, and, finally, control - as well as reduce - the amount of e-mail contacts.

\section{Keywords}

Interpersonal communication, information technology, Internet, e-mail, interaction overload, forms of defense. 


\section{Sumário}

1 Introdução

2. A Comunicação Interpessoal 16

2.1 A comunicação interpessoal offline 16

2.1.1 Os primórdios da comunicação interpessoal 17

2.1.2 A tecnologia nos dispositivos comunicacionais 19

2.2 A revolução das tecnologias da informação 21

2.2.1 Lévy e a revolução virtual 22

2.2.2 Castells e a revolução das tecnologias de informação 24

2.3 A comunicação interpessoal online 25

2.3.1 Origens da comunicação mediada por computadores 30

2.3.2 Tipos de comunicação interpessoal online 32

2.3.2.1 Comunicação síncrona na Internet 32

2.3.2.2 Comunicação assíncrona na Internet 33

2.3.3 A comunicação interpessoal online para Lévy 35

2.3.4 A comunicação interpessoal online para Castells 37

2.3.5 O correio eletrônico 39

2.3.6 Os prós e os contras da comunicação online 43

3 Excessos na Era da Internet 45

3.1 Um breve retorno ao século XVIII 46

$\begin{array}{ll}3.2 \text { Excesso de informação } & 47\end{array}$

3.2.1 A busca desenfreada por informação 50

3.2.2 A oferta de informação não requisitada 52

3.3 Excesso de interação 55 
3.3.1 O contexto da interação

3.3.2 A modalidade da interação 60

3.4 O aumento da demanda de respostas 63

3.5 Defesas tecnológicas $\quad 64$

3.5.1 Alguns recursos para lidar com o excesso de informação 65

3.5.2 Alguns recursos para lidar com o excesso de interação 66

3.6 Defesas psicológicas $\quad 69$

4 Interagindo com os Usuários $\quad 71$

$\begin{array}{ll}4.1 \text { Objetivo } & 71\end{array}$

$\begin{array}{ll}4.2 \text { Sujeitos } & 72\end{array}$

4.3 Instrumento de coleta dos dados $\quad 72$

$\begin{array}{ll}\text { 4.4 Procedimento } & 74\end{array}$

$\begin{array}{ll}\text { 4.5 Análise dos dados } & 75\end{array}$

$\begin{array}{ll}\text { 4.6 Resultados } & 75\end{array}$

$\begin{array}{ll}\text { 4.6.1 Perfil dos sujeitos } & 75\end{array}$

4.6.2 Considerações sobre o correio eletrônico 77

4.6.2.1 A primeira experiência 78

4.6.2.2 Mudanças no uso com o passar do tempo 81

4.6.3.3 Para que usam o e-mail atualmente 83

4.6.3 O dia-a-dia no uso do correio eletrônico 85

4.6.3.1 As expectativas geradas pelo uso 85

4.6.3.2 Hábitos criados $\quad 86$

4.6.3.3 A importância das interações via e-mail 91

4.6.4 Vantagens e considerações positivas sobre o e-mail 94

4.6.4.1 A comunicação antes do e-mail 94 
4.6.4.2 Mudanças trazidas para a vida pessoal dos entrevistados

4.6.5 Desvantagens e considerações negativas 99

4.6.6 O aumento das possibilidades de interação na visão dos 103 usuários

4.6.6.1 Investigando o excesso de interação 103

4.6.6.2 Investigando o excesso da demanda de interações 106

4.6.6.3 A sensação de sobrecarga de interações 110

4.6.7 O que fazer com o aumento das possibilidades de interação 114 e suas conseqüências

5 A Era dos Novos Excessos e Defesas 121

5.1 Principais características do uso do e-mail 121

5.2 A revolução na comunicação interpessoal 125

5.3 Os excessos interativos e os novos mecanismos de defesa 129

5.4 Como lidar com a era dos excessos 132

6 Conclusão 135

7 Referências Bibliográficas 137

8 Bibliografia 140

9 Anexos 142 